\title{
Low temperature Raman scattering study of barium doped lead ytterbium tantalate ceramics
}

\author{
Dibyaranjan Rout $^{*} \S$, Swetapadma Praharaj*, V. Subramanian ${ }^{\dagger}$ and V. Sivasubramanian* \\ *Department of Physics, School of Applied Sciences, KIIT University \\ Bhubaneswar-751 024, Odisha, India \\ ${ }^{\dagger}$ Department of Physics, Indian Institute of Technology, Madras Chennai-600036, India \\ Materials Science Division, IGCAR, Kalpakkam-603102, India

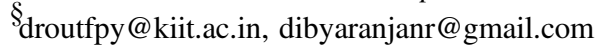

\begin{abstract}
Received 13 September 2016; Revised 29 December 2016; Accepted 29 December 2016; Published 2 February 2017
Raman spectra of a complex perovskite system $\left(\mathrm{Pb}_{1-x} \mathrm{Ba}_{x}\right)\left(\mathrm{Yb}_{05} \mathrm{Ta}_{05}\right) \mathrm{O}_{3}, x=0.0,0.1$ and 0.2 have been studied at low temperature in the range of $10-250 \mathrm{~K}$. The fascination of the system is that it undergoes a crossover from a highly ordered antiferroelectric $(x=0.0)$ to ferroelectric $(x=0.1)$ to disordered relaxor ferroelectric $(x=0.2)$. The nature of splitting that prevails in $\mathrm{F}_{2 \mathrm{~g}}\left(\approx 60 \mathrm{~cm}^{-1}\right), \mathrm{F}_{1 \mathrm{~g}}\left(\approx 538 \mathrm{~cm}^{-1}\right)$ modes and their gradual merging and disappearance with $x$ indicates an orthorhombic symmetry for $x=0.0$ and a pseudo-cubic structure for $x=0.2$. The mode at $420 \mathrm{~cm}^{-1}$ suggests the existence of rhombohedral distortion in all the compositions. The soft mode behavior of the $\mathrm{F}_{2 \mathrm{~g}}$ modes has been analyzed by fitting the data with the power law $\omega_{s}=A\left(T_{C}-T\right)^{\beta}$.

Keywords: Perovskite oxides; B-site ordering; Raman scattering; soft mode.
\end{abstract}

\section{Introduction}

Since last four decades, relaxor ferroelectrics (RFEs) with complex perovskite structure $A\left(B^{\prime} B^{\prime \prime}\right) \mathrm{O}_{3}$ have been extensively studied owing to their potential applications and fascinating basic Physics. ${ }^{1-9}$ The characteristic properties of RFEs include (i) high dielectric constant, (ii) strong dielectric dispersion around $T_{m}$, the temperature corresponding to the dielectric constant maximum, (iii) diffused phase transition around $T_{m}$ and (iv) absence of macroscopic polarization far below $T_{m}$. The polar nanoregions (PNRs) exhibited in RFEs are believed to be responsible for the multi-scale dynamics and spatial in-homogeneity that leads to the above peculiar properties. Generally, RFEs are nonpolar paraelectrics at high temperature, upon cooling PNRs start appearing at $T_{B}$; the socalled Burn temperature and then grow with further decreasing temperature. Nevertheless, when the temperature lowers down to a temperature named freezing temperature $\left(T_{f}\right)$, PNRs become large enough resulting their dynamics to slow down to an extreme point and get frozen into a glassy phase i.e., nonergodic relaxor phase. In some earlier reports, the existence of PNRs in RFEs has already been verified experimentally ${ }^{9-14}$ and also explained the mechanism behind their formation employing different models. ${ }^{14-18}$ However, a conclusive understanding of the physics defining RFEs is still lacking and needs further attention.

In viewpoint of lattice dynamics in displacive ferroelectrics, it has been inferred that the phase transitions are caused by softening and condensation of transverse optic
(TO) phonon modes at zone center. The previous studies demonstrate that in RFE, the dispersion of the transverse acoustic (TA) and low-energy TO phonons at temperatures above $T_{B}$ were noticed to be identical to that observed in the PE phase of normal displacive ferroelectrics. Moreover, the optic mode exhibited in RFEs softens in a similar manner mostly seen in displacive type ferroelectrics when the temperature approaches $T_{B}$ and follows the Cochran law $\omega_{s}=A(T c-T)^{\beta}$. Further, the TO modes in RFEs at $T<T_{f}$ behave consistently with the distinctive behavior of a ferroelectric soft mode. However, in the temperature range between $T_{B}$ and $T_{f}$, the lattice dynamics of RFEs are different from normal ferroelectrics. ${ }^{19-22}$

Using Raman scattering, many groups have studied the soft mode behavior of a displacive or a RFE separately and distinctively. In this present work, an interesting solid solution $\left(\mathrm{Pb}_{1-x} \mathrm{Ba}_{x}\right)\left(\mathrm{Yb}_{0.5} \mathrm{Ta}_{0.5}\right) \mathrm{O}_{3}$ (PBYT) is considered for investigation which undergoes a crossover in phase transition from antiferroelectric $(x=0.0)$ to ferroelectric with diffuse transition $(x=0.1)$ and then to RFE $(x=0.2)$. In the literature, it is also mentioned that the composition $x=0.0$ exhibited weak relaxor behavior around the antiferroelectric to ferroelectric transition. ${ }^{9}$ In fact, the virgin composition $x=$ 0.0 is highly ordered but the degree of ordering decreases with increasing $x .^{23,24}$ Although the degree of ordering is verified by room temperature XRD and Raman spectra, till date, the low temperature Raman study has not been done. As the $T_{m}$ values shifted to lower temperature, it may be

This is an Open Access article published by World Scientific Publishing Company. It is distributed under the terms of the Creative Commons Attribution 4.0 (CC-BY) License. Further distribution of this work is permitted, provided the original work is properly cited. 
interesting to study the Raman spectroscopy at lower temperature to acquire useful information. This study focuses on the Raman spectra of the above mentioned three compositions exhibiting different degree of ordering and phase transition behavior. Emphasis has been given to the phonon dynamics involved in those samples below the freezing temperature $T_{f}$ and hence the Raman spectra were recorded at a very low temperature range $(250-10 \mathrm{~K})$.

\section{Experimental}

The $\left(\mathrm{Pb}_{1-x} \mathrm{Ba}_{x}\right)\left(\mathrm{Yb}_{0.5} \mathrm{Ta}_{0.5}\right) \mathrm{O}_{3}, x=0.0,0.1$ and 0.2 ceramics were prepared by solid-state reaction with optimized calcination and sintering temperatures of $900^{\circ} \mathrm{C}$ and $1325^{\circ} \mathrm{C}$ for $4 \mathrm{~h}$ respectively. ${ }^{9}$ The Raman spectra were taken in the frequency range $1000-40 \mathrm{~cm}^{-1}$ in the low temperature range $250-10 \mathrm{~K}$ and recorded in back scattering geometry using $200 \mathrm{~mW}$ output power of $488 \mathrm{~nm}$ line of an Ar-ion laser. The scattered light was analyzed using a double monochromator (SPEX 14018) and detected with a photo-multiplier tube (Hamamatsu R120) operating in a photon counting mode. For recording Raman spectra at low temperature, a Linkam stage was used along with a $20 \times$ objective. The position and full width at half maximum (FWHM) of the Raman peaks were obtained by fitting the spectra with Lorentzian line shape (Jandel peak fit program). ${ }^{24}$ For each spectra, the number of lines were chosen to obtain a best fit.

\section{Results and Discussion}

Figure 1 shows the Raman spectra of PBYT ceramics at $10 \mathrm{~K}$ in the range $40-1000 \mathrm{~cm}^{-1}$. The number of modes are deconvoluted as 44, 23 and 15 (approximately) for $x=$ $0.0,0.1$ and 0.2 , respectively, and it clearly suggests a structural change with increasing $x$. As per the earlier report, ${ }^{23}$ the

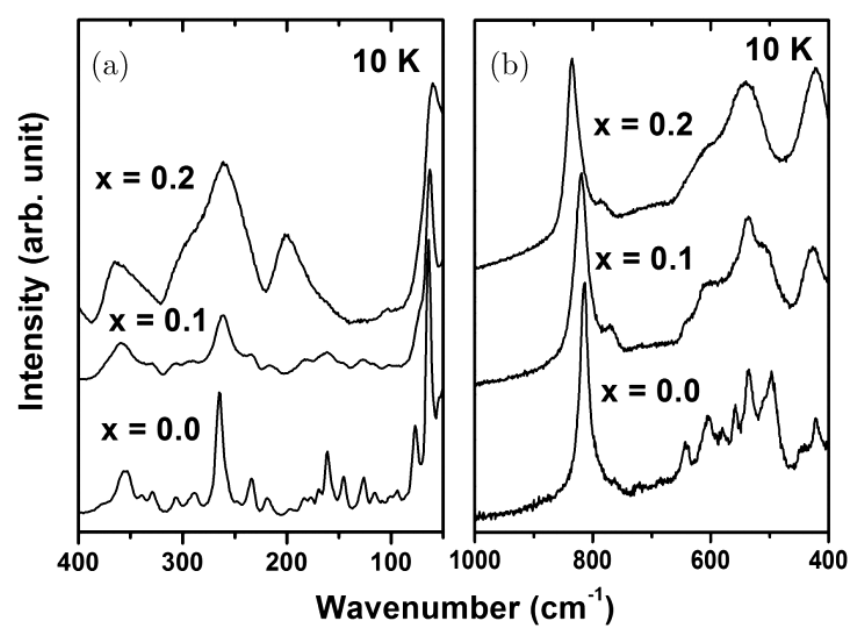

Fig. 1. Raman spectra of $\left(\mathrm{Pb}_{1-x} \mathrm{Ba}_{x}\right)\left(\mathrm{Yb}_{0.5} \mathrm{Ta}_{0.5}\right) \mathrm{O}_{3}$ ceramics at $10 \mathrm{~K}$. structure of PBYT changes from orthorhombically distorted pseudo-monoclinic cell (space group Pbnm) at $x=0.0$ to a pseudo-cubic (space group $F m \overline{3} m$ ) for $x=0.2$. Again, there is possibility of decrease of B-site ordering leading to the disappearance of splitting and subsequently merging and broadening of modes. Further, the number of modes observed for $x=0.2$ is more than the number $\left(4: \mathrm{A}_{1 \mathrm{~g}}+\mathrm{E}_{\mathrm{g}}+2 \mathrm{~F}_{2 \mathrm{~g}}\right)$ predicted theoretically for $F m \overline{3} m .^{25}$ This indicates a deviation of the local structure from the average structure due to the presence of PNRs as the system exhibited relaxor behavior. Nevertheless, the contribution of phase coexistence and distortion cannot be brushed aside because the composition $x=$ 0.2 is suggested as the morphotropic phase boundary for the present solid solution. ${ }^{24}$ Figure 2 plots the temperature dependence of Raman spectra for all the ceramics in the frequency range $40-100 \mathrm{~cm}^{-1}$. In this frequency range, three strong peaks are observed for $x=0.0$ and one broad peak for $x=0.2$. The mode observed around $60 \mathrm{~cm}^{-1}$ is assigned to the $\mathrm{F}_{2 \mathrm{~g}} \mathrm{~Pb}$-localized mode. Generally, in complex perovskites, $F_{2 g}$ mode arises due to the movement of A-site cations along with the oxygen atoms and the position of the mode expected to be influenced by the mass of A cation and A-O bond parameters. ${ }^{26}$

As per theoretical calculation, in cubic symmetry, the $F_{2 g}$ modes are triply degenerate but when the symmetry reduces to orthorhombic, these modes split into three lines. Our results show excellent matching to the theoretical prediction as the composition, $x=0.2$ with pseudo-cubic symmetry display a single broad peak and subsequently split into three peaks around the peak at $60 \mathrm{~cm}^{-1}$ for the composition $x=$ 0.0 exhibiting orthorhombic symmetry. Again, it appears that there is a small shift observed for $F_{2 g}$ towards lower frequency with $\mathrm{Ba}$ concentration as anticipated because the mass of $\mathrm{Pb}$ is more.

Nevertheless, a careful study of this mode as a function of temperature reveals a reasonable softening of the mode and can be assigned to the soft mode in this system. Temperature dependence of the soft mode wave number is plotted in Fig. 3 for all the three compositions. Figure 3(a) is reproduced to carry out a comparative study.

The soft modes show a nonlinear variation and fit into the power relation $\omega_{s}=A\left(T_{C}-T\right)^{\beta}$, where $\omega_{s}$ is the mode frequency, $A$ is a constant, $T_{C}$ is the expected ferroelectric phase transition temperature and $\beta$ is a critical exponent related to order parameter. ${ }^{27}$ Actually, this PBYT system is a very interesting system. As per the temperature-dependent dielectric data, PYT exhibits weak relaxor behavior at lower temperature, marked by transition from antiferroelectric to ferroelectric state. ${ }^{24}$ With increasing $\mathrm{Ba}$, the relaxor behavior becomes stronger. Generally, it is considered that the relaxor phase transition is not a first-order phase transition rather it is likely to be a second-order phase transition. ${ }^{28}$ Moreover, the optic mode exhibited in RFEs softens in a similar manner mostly seen in displacive type ferroelectrics when the temperature approaches $T_{B}$ and follows the Cochran law. ${ }^{27}$ 


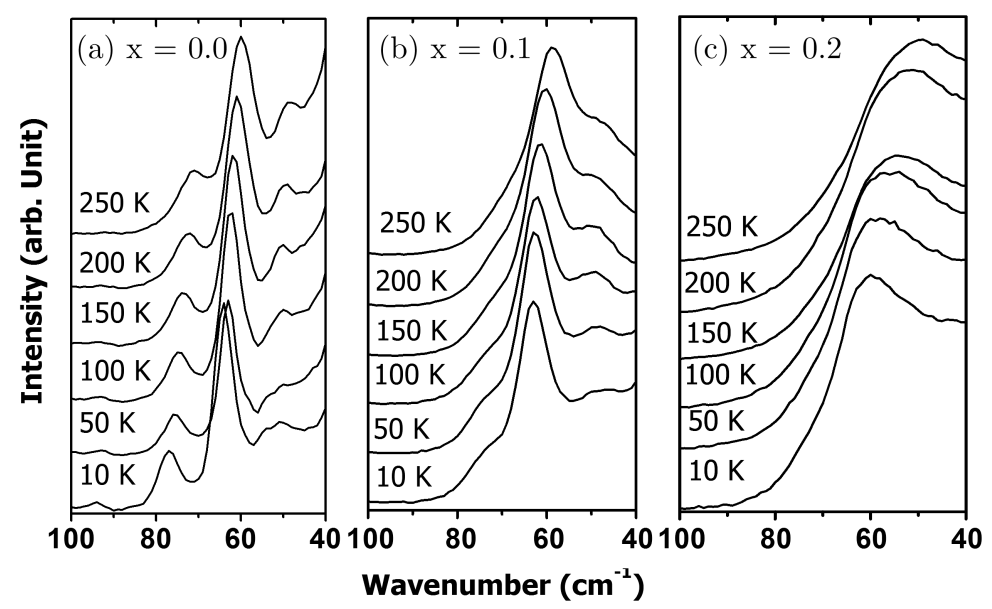

Fig. 2. Temperature-dependent Raman spectra in the frequency range $100-30 \mathrm{~cm}^{-1}$.

Further, the TO modes in RFEs at $T<T_{f}$ behave consistently with the distinctive behavior of a ferroelectric soft mode. ${ }^{22} \mathrm{In}$ analogy to the above facts, we have used Cochran law $\omega_{s}=$ $A(T c-T)^{\beta}$ to analyze the soft mode for the system.

In Fig. 3, the experimental data are shown by scattered points and the solid lines are the fitted curves to the above mentioned power law. The fitting parameters are given in the figure. The fitting parameters indicate a gradual increase of constant $A$ and a gradual decrease of the critical exponent $\beta$ as $\mathrm{Ba}$ concentration increases. This decrease of $\beta$ may be related to the gradual crossover from first-order phase transition to diffuse transition then to weak relaxor behavior. It can be inferred that the degree of interaction between the PNRs and their freezing temperatures are different in those compositions, which may directly or indirectly influence the phonon dynamics. Moreover, the $T_{c}$ values $426 \mathrm{~K}(x=0.0)$, $390 \mathrm{~K}(x=0.1)$ and $283 \mathrm{~K}(0.2)$ obtained from the fitting are in close accordance with the ferroelectric phase transition temperatures reported earlier in dielectric constant versus temperature data for the compositions respectively. ${ }^{9,24}$ Hence, the possibility of a significant contribution of the soft mode in the ferroelectric phase transition in all the compositions cannot be avoided.

Figure 4 shows the Raman spectra in the range 400$100 \mathrm{~cm}^{-1}$ recorded at different temperatures. In this frequency range, three major peaks at $\approx 200,259$ and $360 \mathrm{~cm}^{-1}$ are observed at $x=0.2$, but with reduction of symmetry at lower compositions, each one of the peak clearly split into three peaks and several new peaks appeared. The broad peak around $200 \mathrm{~cm}^{-1}$ with a hump around $177 \mathrm{~cm}^{-1}$ emerges due to the relative rotation of octahedron due to size mismatch in $\mathrm{B}^{\prime}-\mathrm{O}-\mathrm{B}^{\prime \prime}$ bond and attributed to $\mathrm{F}_{1 \mathrm{~g}}$ mode. The other two strong peaks at $\approx 259$ and $360 \mathrm{~cm}^{-1}$ attributed to $\mathrm{F}_{1 \mathrm{u}}$ mode due to asymmetric $\mathrm{O}-\mathrm{B}-\mathrm{O}$ bending vibration arising from rhombohedral structural distortion and $\mathrm{F}_{2 \mathrm{u}}$ mode due to electron-phonon coupling of $\mathrm{Pb}^{2+}$ lone pair electrons respectively. The changes noticed in those modes further evidence the orthorhombic symmetry along with rhombohedral distortion in lower concentration; with increasing $\mathrm{Ba}$ concentration, the structure gradually modifies towards higher symmetry but the rhombohedral symmetry remains present within the studied compositions. Moreover, with increasing temperature, these modes undergo no substantial shift in their wave number but as expected, they show thermal broadened. The presence of an asymmetric $\mathrm{O}-\mathrm{B}-\mathrm{O}$ bending vibration mode at $420 \mathrm{~cm}^{-1}$ (Fig. 5) with $\mathrm{F}_{1 \mathrm{u}}$ symmetry provide further evidences to confirm the existence of rhombohedral distortion. ${ }^{29}$ In fact, the mode evolves as stronger and prominent as the $\mathrm{Ba}$ concentration increases. It remains to be stronger with increasing temperature
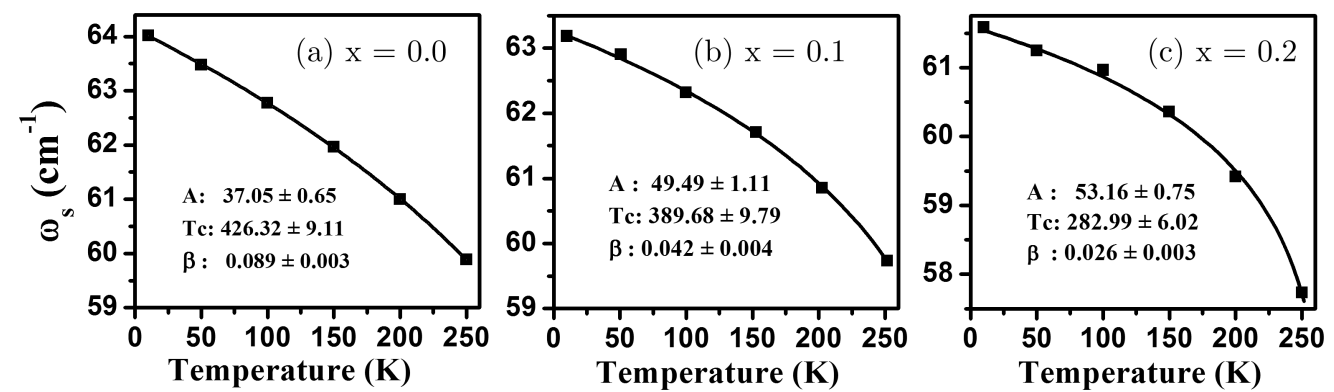

Fig. 3. Temperature-dependent of frequency of $\mathrm{F}_{2 \mathrm{~g}}$ mode at $60 \mathrm{~cm}^{-1}$. The scatter point are the experimental data and the solid line is the fitting curve to the power relation. 


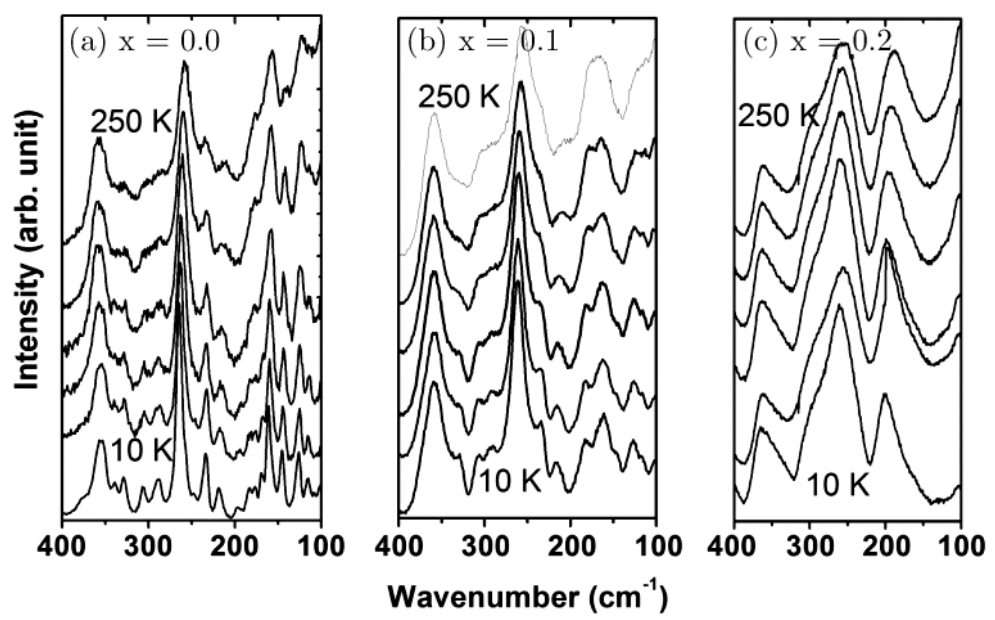

Fig. 4. Temperature-dependent Raman spectra in the frequency range $400-100 \mathrm{~cm}^{-1}$.

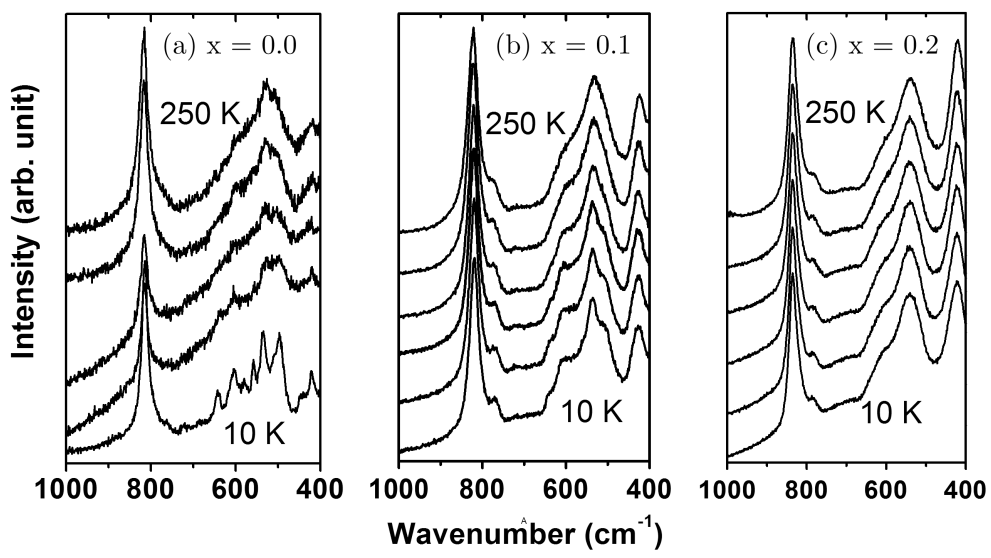

Fig. 5. Temperature-dependent Raman spectra in the frequency range $1000-400 \mathrm{~cm}^{-1}$.

suggesting the persistence of rhombohedral symmetry in those samples, which is one of the reasons for the increase in diffuseness. Hence, the present observations are considerably in accordance with the earlier prediction of a morphotropic phase boundary i.e., the coexistence of more than one structural phases at the composition $x=0.2$ as per the dielectric study.

Figure 5 plots the Raman spectra in the frequency range $1000-400 \mathrm{~cm}^{-1}$ and displays two strong and characteristic peaks at $\approx 535 \mathrm{~cm}^{-1}$ and $835 \mathrm{~cm}^{-1}$. The former is assigned to $\mathrm{F}_{2 \mathrm{~g}}$ symmetric bending of $\mathrm{BO}_{6}$ octahedra and later is assigned to the $A_{1 g}$ mode arises due to the movement of oxygen atoms along $\mathrm{B}^{\prime}-\mathrm{O}-\mathrm{B}^{\prime \prime}$ with cations at rest. Usually, this high frequency $\mathrm{A}_{1 \mathrm{~g}}$ mode is a measure of $\mathrm{B}$-site ordering in complex perovskites. ${ }^{26}$ The shifting of this mode indicates a change in the degree of ordering due to the size mismatch at $\mathrm{A}$-site as $\mathrm{Pb}$ is substituted by $\mathrm{Ba}$ in this present system. In analogy to the earlier dielectric studies, the B-site ordering decreases with increasing $\mathrm{Ba}$ concentration. However, the mode undergoes no change with increasing temperature maintaining the short range ordering in the temperature range studied. Nevertheless, the high frequency $F_{1 \mathrm{~g}}$ mode shifts towards lower frequency side with increasing temperature. Though the shift is observed in all the samples, the level of shifting gradually decreases with Ba concentration. This may be due to the fact that the FE phase transitions are gradually showing relaxor type behavior in Ba substituted compounds though at low temperatures all the samples exhibit FE phase. Also, there is possible modification in antiferroelectric coupling of $\mathrm{Pb}^{2+}$ cations and ferroelectric coupling of B-site cations as the system crossover from antiferroelectric to ferroelectric and then to RFE with increasing $\mathrm{Ba}$ concentration.

\section{Summary}

Single phase $\left(\mathrm{Pb}_{1-x} \mathrm{Ba}_{x}\right)\left(\mathrm{Yb}_{0.5} \mathrm{Ta}_{0.5}\right) \mathrm{O}_{3}, x=0.0,0.1$ and 0.2 ceramics were prepared by solid state reaction method and Raman spectra were recorded on those samples in the frequency range $1000-40 \mathrm{~cm}^{-1}$ at low temperatures $250-10 \mathrm{~K}$. The spectra revealed orthorhombic symmetry for the antiferroelectric PYT and the structure gradually changed to a pseudo-cubic symmetry. The existence of rhombohedral 
distortion was also evidenced in the PYT sample and the distortion further increased with increase of Ba concentration. The low frequency $\mathrm{F}_{2 \mathrm{~g}}$ mode at $\approx 60 \mathrm{~cm}^{-1}$ showed soft mode behavior in all compositions suggesting ferroelectric phase at low temperature. The mode clearly fit into a power law and the $T_{c}$ values obtained from each fit match well with the phase transition temperature observed in dielectric measurements of those samples.

\section{References}

${ }^{1}$ L. E. Cross, Relaxor ferroelectrics, Ferroelectrics 76, 241 (1987).

${ }^{2} \mathrm{H}$. Fu and R. E. Cohen, Polarization rotation mechanism for ultrahigh electromechanical response in single-crystal piezoelectrics, Nature 403, 281 (2000).

${ }^{3}$ A. A. Bokov and Z.-G. Ye, Recent progress in relaxor ferroelectrics with perovskite structure, Front. Ferroelectricity 41, 31 (2006).

${ }^{4}$ B. Mihailova, B. Maier, T. Steilmann, E. Dul'kin and M. Roth, Electric-field-induced local structural phenomena in $\mathrm{Pb}$-based $\mathrm{ABO}_{3}$-type relaxor ferroelectrics, IEEE Trans. 62, 7 (2015).

${ }^{5}$ D. J. Goossens, Local ordering in lead-based relaxor ferroelectrics, Acc. Chem. Res. 46, 2597 (2013).

${ }^{6}$ V. V. Shvartsman and D. C. Lupascu, Lead-free relaxor ferroelectrics, J. Am. Ceram. Soc. 95, 1 (2012).

${ }^{7}$ G. Burns and F. H. Dacol, Crystalline ferroelectrics with glassy polarization behavior, Phys. Rev. B 28, 2527 (1983).

${ }^{8}$ Z. Trybuła, S. Miga, S. Łoś, M. Trybuła and J. Dec, Evidence of polar nanoregions in quantum paraelectric $\mathrm{KTaO}_{3}$, Solid State Commun. 209, 23 (2015).

${ }^{9}$ D. Rout, V. Subramanian, K. Hariharan, V. Sivasubramanian and V. R. K. Murthy, Dielectric study of the phase transitions in $\left(\mathrm{Pb}_{1-x} \mathrm{Ba}_{x}\right)\left(\mathrm{Yb}_{1 / 2} \mathrm{Ta}_{1 / 2}\right) \mathrm{O}_{3}$ ceramics, Ferroelectrics 300, 67 (2004).

${ }^{10}$ C. Stock, P. M. Gehring, H. Hiraka, I. Swainson, G. Xu, Z. G. Ye, H. Luo, J. F. Li and D. Viehland, Evidence for anisotropic polar nanoregions in relaxor $\mathrm{Pb}\left(\mathrm{Mg}_{1 / 3} \mathrm{Nb}_{2 / 3}\right) \mathrm{O}_{3}$ : A neutron study of the elastic constants and anomalous TA phonon damping in PMN, Phys. Rev. B 86, 104108 (2012).

${ }^{11} \mathrm{~F}$. Li, Z. Xu and S. Zhang, The effect of polar nanoregions on electromechanical properties of relaxor- $\mathrm{PbTiO}_{3}$ crystals: Extracting from electric-field-induced polarization and strain behaviors, Appl. Phys. Lett. 105, 122904 (2014).

${ }^{12}$ K. Hirota, Z. G. Ye, S. Wakimoto, P. M. Gehring and G. Shirane, Neutron diffuse scattering from polar nanoregions in the relaxor $\mathrm{Pb}\left(\mathrm{Mg}_{1 / 3} \mathrm{Nb}_{2 / 3}\right) \mathrm{O}_{3}$, Phys. Rev. B 65, 104105 (2002).

${ }^{13}$ I. K. Jeong, T. W. Darling, J. K. Lee, T. Proffen, R. H. Heffner, J. S. Park, K. S. Hong, W. Dmowski and T. Egami, Direct observation of the formation of polar nanoregions in $\mathrm{Pb}\left(\mathrm{Mg}_{1 / 3} \mathrm{Nb}_{2 / 3}\right)$ $\mathrm{O}_{3}$ using neutron pair distribution function analysis, Phys. Rev. Lett. 94, 147602 (2005).
${ }^{14}$ M. E. Manley, J. W. Lynn, D. L. Abernathy, E. D. Specht, O. Delaire, A. R. Bishop, R. Sahul and J. D. Budai, Phonon localization drives polar nanoregions in a relaxor ferroelectric, Nature Commun. 5, 3683 (2014).

${ }^{15}$ W. Kleemann, J. Dec and S. Miga, The cluster glass route of relaxor ferroelectrics, Phase Transitions 88, 234 (2015).

${ }^{16}$ B. E. Vugmeister and H. Rabitz, Dynamics of interacting clusters and dielectric response in relaxor ferroelectrics, Phys. Rev. B 57, 7581 (1998).

${ }^{17}$ R. Pirc and R. Blinc, Spherical random-bond-random-field model of relaxor ferroelectrics, Phys. Rev. B 60, 13470 (1999).

${ }^{18}$ V. Westphal, W. Kleemann and M. D. Glinchuk, Diffuse phase transitions and random-field-induced domain states of the relaxor ferroelectric $\mathrm{PbMg}_{1 / 3} \mathrm{Nb}_{2 / 3} \mathrm{O}_{3}$, Phys. Rev. Lett. 68, 847 (1992).

${ }^{19} \mathrm{~K}$. Tani, Dynamics of displacive-type ferroelectrics-soft modes, $J$. Phys. Soc. Japan 26, 93 (1969).

${ }^{20}$ A. Nabereznov, S. Vakhrushev, B. Dorner, D. Strauch and H. Moudden, Inelastic neutron scattering study of the relaxor ferroelectric $\mathrm{PbMg}_{1 / 3} \mathrm{Nb}_{2 / 3} \mathrm{O}_{3}$ at high temperatures, Eur. Phys. $J$. B-Condens. Matter Complex Syst. 11, 13 (1999).

${ }^{21}$ P. M. Gehring, S. Wakimoto, Z.-G. Ye and G. Shirane, Soft mode dynamics above and below the burns temperature in the relaxor $\mathrm{Pb}$ $\left(\mathrm{Mg}_{1 / 3} \mathrm{Nb}_{2 / 3}\right) \mathrm{O}_{3}$, Phys. Rev. Lett. 87, 277601 (2001).

${ }^{22}$ S. Wakimoto, C. Stock, R. J. Birgeneau, Z.-G. Ye, W. Chen, W. J. L. Buyers, P. M. Gehring and G. Shirane, Ferroelectric ordering in the relaxor $\mathrm{Pb}\left(\mathrm{Mg}_{1 / 3} \mathrm{Nb}_{2 / 3}\right) \mathrm{O}_{3}$ as evidenced by low-temperature phonon anomalies, Phys. Rev. B 65, 172105 (2002).

${ }^{23}$ D. Rout, V. Subramanian and V. Sivasubramanian, Temperature dependence Raman spectroscopic studies of $\mathrm{Pb}\left(\mathrm{Yb}_{0.5} \mathrm{Ta}_{0.5}\right) \mathrm{O}_{3}$, Vib. Spectrosc. 46, 22 (2008).

${ }^{24}$ D. Rout, V. Subramanian, K. Hariharan, V. Sivasubramanian and V. R. K. Murthy, Raman spectroscopic study of $\left(\mathrm{Pb}_{1-x} \mathrm{Ba}_{x}\right)$ $\left(\mathrm{Yb}_{1 / 2} \mathrm{Ta}_{1 / 2}\right) \mathrm{O}_{3}$ ceramics, J. Appl. Phys. 98, 103503 (2005).

${ }^{25}$ U. Bismayer, V. Devarajan and P. Groves, Hard-mode Raman spectroscopy and structural phase transition in the relaxor ferroelectric lead scandium tantalate, $\mathrm{Pb}\left(\mathrm{Sc}_{0.5} \mathrm{Ta}_{0.5}\right) \mathrm{O}_{3}$, J. Phys. Condens. Matter 1, 6977 (1989).

${ }^{26}$ I. G. Siny, R. Tao, R. S. Katiyar, R. Guo and A. S. Bhalla, Raman spectroscopy of $\mathrm{Mg}$-Ta order-disorder in $\mathrm{BaMg}_{1 / 3} \mathrm{Ta}_{2 / 3} \mathrm{O}_{3}, J$. Phys. Chem. Solids 59, 181 (1998).

${ }^{27}$ A. Hushur, Y. Akishige and S. Kojima, Soft optical phonon in Badoped $\mathrm{Sr}_{2} \mathrm{Nb}_{2} \mathrm{O}_{7}$ ceramics, Ceram. Int. 30, 2023 (2004).

${ }^{28}$ G. A. Samara, The relaxational properties of compositionally disordered $\mathrm{ABO}_{3}$ perovskites, J. Phys.: Condens. Matter 15, R367 (2003).

${ }^{29}$ B. Mihailova, U. Bismayer, B. Guttler, M. Gospodinov and L. Konstantinov, Local structure and dynamics in relaxor-ferroelectric $\mathrm{PbSc}_{1 / 2} \mathrm{Nb}_{1 / 2} \mathrm{O}_{3}$ and $\mathrm{PbSc}_{1 / 2} \mathrm{Ta}_{1 / 2} \mathrm{O}_{3}$ single crystals, $J$. Phys: Condens. Matter 14, 1091 (2002). 